caused the sudden catastrophic collapse. A similar deterioration to ventricular fibrillation has been reported 70 hours after ingestion. ${ }^{6}$ The plasma flecainide concentration at 73 hours in this patient was $0.7 \mathrm{mg} / \mathrm{l}$ having been $6.5 \mathrm{mg} / 1$ three hours after ingestion. This patient however, responded to a single defibrillatory shock. A second patient reported by the same author deteriorated to an electromechanical dissociation cardiac arrest 6.5 hours after flecainide overdose despite recording a blood pressure of $140 / 80 \mathrm{~mm} \mathrm{Hg}$ before the arrest. Resuscitation was unsuccessful in this case.

Acute overdose of flecainide acetate is rare but produces serious cardiac compromise. The primary treatment aim is to prevent drug absorption by gastric lavage and administration of activated charcoal. Secondary measures to prevent cardiac conduction disturbance include the use of a prophylactic pacemaker and inotropic drugs. Attempts to displace the sodium channel blocking drug by administration of hypertonic sodium bicarbonate require frequent electrolyte measurements. Invasive techniques of haemodynamic support may allow clearance and redistribution to occur. We would recommend that all patients with flecainide overdose be admitted to intensive care for invasive monitoring even if they appear clini- cally stable as they are at risk of subsequent deterioration.. Despite these measures, however, mortality remains high.

1 Rogers C, Anderson DT, Ribe JK, et al. Fatal flecainide intoxication. J Anal Toxicol 1993;17:434-5.

2 Yasui RK, Culclasure TF, Kaufman D, et al. Flecainide overdose: is cardiopulmonary support the treatment ? Ann Emerg Med 1997;29:680-2.

3 Winkelmann BR, Leinberger $\mathrm{H}$. Life-threatening flecainide toxicity. A pharmacodynamic approach. Ann Intern Med 1987;106:807-14.

4 Kjekshus J, Bathen J, Orning OM, et al. A double-blind crossover comparison of flecainide acetate and disopyramide phosphate in the treatment of ventricular premature complexes. Am J Cardiol 1984;53:72B-8B.

5 Duff $\mathrm{HJ}$, Roden DM Maffucci RJ, et al Suppression of resistant ventricular arrhythmias by twice daily dosing with resistant ventricular arrhythmias by twice dail

6 Gotz D, PohleS, Barckow D. Primary and secondary detoxification in severe flecainide intoxication. Intensive Care Med 1991;17:181-4.

7 Wurzberger R, Witter E, Avenhaus $\mathrm{H}$, et al. Haemoperfusion bie Flecainidintoxikation. Klin Wochenschr 1986;64: $442-4$

8 Salerno DM, Murakami MM, Johnston RB, et al. Reversal of flecainide-induced ventricular arrhythmia by hypertonic sodium bicarbonate in dogs. Am J Emerg Med 1995;13: 285-93.

9 Bou-Abboud E, Nattel S. Relative role of alkalosis and sodium ions in reversal of class 1 antiarrhythmic drug induced sodium channel blockade by sodium bicarbonate. Circulation 1996;94:1954-61.

10 Ranger S, Sheldon R, Fermini B, et al. Modulation of flecainide's cardiac sodium blocking actions by extracellular nide's cardiac sodium blocking actions by extracellular sodium: a possible cellular mechanism for the action of sodium salts in flecainide

11 Greig J, Groden BM. Persistent electrocardiographic changes after flecainide overdose. Br J Clin Pract 1995;49: changes

12 Overlie PA. Emergency use of cardiopulmonary bypass. Journal of Interventional Cardiology 1995;8:239-47.

\title{
A case of streptococcal myositis (misdiagnosed as hamstring injury)
}

\author{
Norbert Kang, Dimitrios Antonopoulos, Atul Khanna
}

RAFT Institute of

Plastic Surgery, Mount

Vernon Hospital,

Northwood, Middlesex

N Kang

Department of Plastic Surgery, St Andrew's

Hospital, Patras,

Greece

D Antonopoulos

Department of Plastic Surgery, Wordlsey

Hospital, Stourbridge, West Midlands

A Khanna

Correspondence to: Mr Norbert Venantius Kang, 6 Elgin Drive, Northwood, Middlesex HA6 2YR.

Accepted for publication 27 July 1998

\begin{abstract}
Streptococcal myositis is a very rare bacterial infection of muscle with a high mortality. Diagnosis is difficult because of the paucity of clinical signs and symptoms at the onset. However, presentation of the disease appears to have changed over the last 50 years. A case of streptococcal myositis is presented (misdiagnosed as hamstring injury), which more closely reflects the current presentation of the disease. Some of the features that may help emergency clinicians to recognise the onset of the condition are highlighted. (F Accid Emerg Med 1998;15:425-426)
\end{abstract}

Keywords: necrotising fasciitis; myonecrosis; streptococcal myositis

Streptococcal myositis is an acute infection of muscle by invasive group A $\beta$ haemolytic streptococcus causing myonecrosis without abscess formation. It differs from the more frequent and relatively benign streptococcal pyomyositis, which is characterised by the formation of abscesses in muscle and which has a good prognosis.
The standard surgical texts are misleading: "Streptococcal myositis resembles acute clostridial gas gangrene and was not described until World War II. After an incubation period of 3 to 4 days there is swelling, edema, and purulent wound exudate. These signs are followed by pain which rapidly becomes severe. Gas is present and the infected muscle changes from pale and soft to bright red, striped with purple and finally purple and gangrenous. The seropurulent discharge has a sour odour".

The principle source of this description is MacLennan who collected a series of eight cases of streptococcal myositis in soldiers with battle wounds during World War II. ${ }^{2}$ This description differs from more recent reports including our own experience of the condition.

\section{Case report}

A 33 year old labourer presented with 24 hours of increasing pain in the medial aspect of his right thigh. There was no history of recent trauma. Slight erythema, tenderness, and swelling were noted. The pain was worse with movement especially extension and abduction Sensation and pulses were intact throughout the lower limb. He was afebrile. A diagnosis of 


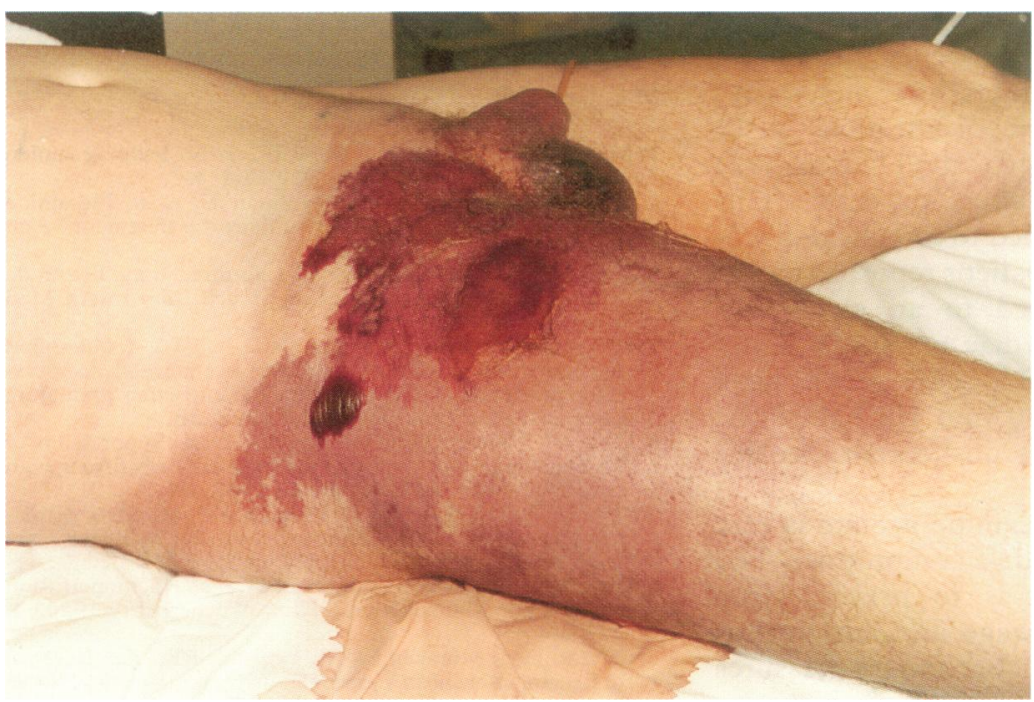

Figure 1 Right thigh with mottling, haemorrhagic bullae, and skin necrosis suggestive of necrotising fasciitis ( 72 hours after onset of symptoms). The underlying streptococcal myositis was not apparent until debridement of the necrotic skin was performed.

hamstring strain was made. He was given naproxen, a support bandage, prescribed a course of physiotherapy, and discharged. He returned the next day with increasing pain in his right thigh and vomiting. The whole of his right lower limb, particularly the thigh, was grossly swollen, and "bruised". An ultrasound scan showed no evidence of deep vein thrombosis, muscle rupture, haematoma or abscess, but did show diffuse oedema of the muscle. Blood results were normal except for urea 13.6 $\mathrm{mmol} / \mathrm{l}$, creatinine $0.228 \mathrm{mmol} / \mathrm{l}$, and creatinine kinase $2491 \mathrm{IU} / 1$. Plain radiographs of the right thigh showed diffuse oedema of muscle but no foreign body and no fracture. Seven hours after admission the patient developed haemorrhagic bullae. He was afebrile, but hypotensive and tachycardic. Ten hours after admission his right thigh, groin, and scrotum became gangrenous (fig 1). At operation (72 hours after the onset of symptoms) the skin of the right lower limb, and right lower quadrant of the abdomen, were necrotic. Because the muscles of the thigh were necrotic, a hip disarticulation was performed. The patient remained in intensive care for four weeks and returned home eight weeks after admission.

\section{Discussion}

In 1994, the Public Health Laboratory Service (PHLS) in the UK initiated a system of enhanced surveillance for all invasive group A streptococcal infections. Between 1 July 1994 and 30 June 1996 there were 1092 reported cases of invasive group $A$ streptococcal infection in England and Wales. Of these, only four were described as invasive group A streptococcal infections of muscle without abscess formation.

Our review of previously published cases of streptococcal myositis and those recently re- ported to the PHLS indicated a male to female ratio of $2: 1 .^{3-7}$ This compares with a male to female ratio of $0.9: 1$ for all invasive group A streptococcal infections. The typical patient was aged 30 to 40 years with no significant previous medical history. A portal of entry for infection was present in only five of previously published cases and none in the four cases recently reported to the PHLS. This compares with the open battle wounds described by MacLennan. ${ }^{2}$ The first symptom was usually increasing pain in the infected muscle. This is in contrast to simple hamstring injury or the description in the surgical texts which describe "swelling, edema, and purulent wound exudate" preceding the onset of pain. The most frequent misdiagnoses were flu/viral illness, deep vein thrombosis, or muscle strain/rupture/ haematoma/abscess. The lower limbs were affected in approximately $50 \%$ of all cases. Skin involvement with haemorrhagic bullae, blue mottling, and ecchymosis were late features. In further contrast to MacLennan, intramuscular gas was not a feature of any recent cases. However, when plain radiographs or ultrasound were performed at initial presentation, oedema of the affected muscle was seen. In addition, serum creatinine kinase levels (where recorded) were always raised at initial presentation.

For the emergency room physician faced with a possible case of streptococcal myositis, the most significant symptom appears to be pain in the infected muscle that worsens rapidly over 24-48 hours. Once the index of suspicion has been raised, ultrasound and plain radiographs will help to distinguish streptococcal myositis from more common diagnoses while a raised serum creatinine kinase will clinch the diagnosis well before streptococci can be cultured. The recommended treatment remains aggressive surgical debridement of all infected tissue with high dose benzylpenicillin (2.4 g, every four hours) plus clindamycin (0.6-1.2 g, every six hours) in severe cases.

I gratefully acknowledge the help of Dr M Monninckendam and Dr R C George in providing data on cases of invasive group $A$ streptococcal infection reported to the PHLS between 1 July 1994 and 30 June 1996.

1 Cohn I, Bornside GH. Infections. In: Schwartz SI, Shire GT, Spencer FC, eds. Principles of surgery. 5th Ed. New York: McGraw Hill, 1989.

2 MacLennan JD. Streptococcal infection of muscle. Lance 1943;i:582-4.

3 Razi-Syed S, Jafri SZ. Necrotizing fasciitis and myositis: case report. Comput Med Imaging Graph 1994;18: 213-16.

4 Hird B, Byrne K. Gangrenous streptococcal myositis; case report. J Trauma 1994;36:589-91.

5 Jahnson L, Berggen L, Biorsell-Ostling E, et al. StreptococJahnson L, Berggen L, Bjorsell-Ostling E, et al. Stre

6 Demey HE, Goovaerts GC, Pattyn SR, et al Streptococcal myositis: a report of 2 cases. Acta Clin Belg 1991;46:82-8. 7 Doebbling BN, Wenzel RP. Spontaneous streptococcal gan7 Doebbling BN, Wenzel RP. Spontaneous streptococcal ganMed J 1989;82:900-2. 